\title{
Article \\ Symplectic Entropy as a Novel Measure for Complex Systems
}

\author{
Min Lei 1,*, Guang Meng 1,*, Wenming Zhang ${ }^{1}$, Joshua Wade ${ }^{2}$ and Nilanjan Sarkar ${ }^{2, *}$ \\ 1 Institute of Vibration, Shock and Noise, State Key Laboratory of Mechanical System and Vibration, \\ Shanghai Jiao Tong University, Shanghai 200240, China; wenmingz@sjtu.edu.cn \\ 2 Mechanical Engineering, Vanderbilt University, Box 1592B, Nashville, TN 37235, USA; \\ joshua.w.wade@vanderbilt.edu \\ * Correspondence: leimin@sjtu.edu.cn (M.L.); gmeng@sjtu.edu.cn (G.M.); \\ nilanjan.sarkar@vanderbilt. edu (N.S.)
}

\begin{abstract}
The real systems are often complex, nonlinear, and noisy in various areas including mathematics, natural science, and social science. We present the symplectic entropy (SymEn) measure as well as an analysis method based on SymEn to estimate the nonlinearity of the complex system by analyzing the given time series. The SymEn estimation is a kind of entropy based on symplectic principal component analysis (SPCA) which represent organized but unpredictable behaviors of systems. The key to SPCA is to preserve the global submanifold geometrical properties of the systems through symplectic transform in the phase space, which is a kind of the measurepreserving transforms. The capability of preserving the global geometrical characteristics makes the SymEn a test statistic to detect the nonlinear characteristics in several typical chaotic time series and the stochastic characteristic in the Gaussian white noise. The results are in agreement with findings in the approximate entropy (ApEn), the sample entropy (SampEn) and the fuzzy entropy (FuzzyEn). Moreover, the SymEn method is also used to analyze the nonlinearities of the real signals (including the EEG signals for ASD and healthy subjects, and the sound and vibration signals for the mechanical systems). The results indicate that the SymEn estimation can be taken as a measure for describing the nonlinear characteristics in the data collected from the natural complex systems.
\end{abstract}

Keywords: symplectic geometry; symplectic principal component analysis; symplectic entropy; complex system.

\section{Introduction}

Complex systems have been widely studied in various fields such as mathematics, physics, engineering, economics, biomedical engineering, and so on. Due to the unknown structures of systems and the complicated and disorder forms of the measured time series, it has been always challenging to explore the nature characteristics of complex systems (such as the brain) [1-2]. The essential problem for a complex system is to differentiate between nonlinearity and linear stochastics because a complex system, especially with chaos, is often very similar to a random process. Symplectic geometry is a geometry in an even dimensional phase space with a closed nondegenerate skew-symmetric bilinear form. Due to the capability of measure-preserving of symplectic similar transform in phase space, symplectic geometry could preserve the system structure, even non-linear structure. The global submanifold geometrical properties in the system or the data from the system can keep unchanged through symplectic transforms in phase space [3-5]. The aim of this study is to employ the measure-preserving characteristics of symplectic geometry to propose an entropy measure for complex systems. The entropy as a statistic measure can distinguish complex systems from linear stochastic processes. Symplectic geometry has been used to study complex systems in the two areas: solving symplectic differential equations for various nonlinear dynamical systems [6-11] and analyzing a time series in phase space geometry for testing 
the nature of complex systems [3-4, 12]. However, there has been little research on the entropy estimation in the symplectic theory.

In fact, the studies and applications of symplectic geometry are mainly to solve symplectic differential systems in mathematics, physics, theoretical and classical mechanics since Feng and his research group first developed symplectic approach to solve accurately some nonlinear dynamical systems constructed using Hamiltonian models [9]. The mathematical fundamentals of symplectic geometry are based on the symplectic space that is different from Euclidean space although the two spaces could deal with the Hamilton system. The symplectic similarities in the symplectic space can preserve the structures of the Hamiltonian matrices [13-15]. Some numerical algorithms based on symplectic geometry, such as symplectic Householder transformations, symplectic QR-like decomposition [16] or symplectic Gram-Schmidt algorithm [17], are proposed and modified to solve the eigenvalues of the Hamiltonian matrices, particularly for sparse and large structured matrices [18]. The symplectic eigensolutions are proposed to perform the energy band analysis for a periodical waveguide by introducing symplectic mathematics into the electro-moagnetic waveguide theory [19]. A novel method based on symplectic mathematics and finite element analysis is developed to deduce the dispersion relations for some typical cellular structures [20]. For some basic problems in solid mechanics and elasticity, many of the research works of symplectic elasticity are attributed to Zhong, $\mathrm{Xu}$, Zhang, Yao, Leung and Lim and their co-workers [9, 20-22]. Subsequently, the symplectic analysis has been applied to solve other applied engineering problems, such as control problems [23], piezoelectric materials [24], bending problems of corner-supported rectangular thin plates [25-26] and so on.

Recently, the symplectic geometry theory has been applied to analyze the time series from complex systems through the reconstructed phase space [4]. According to the Takens' embedding theorem, the reconstructed phase space is equivalent (diffeomorphic) to the attractor of the original dynamical system generating the data so that it can reflect the dynamical characteristics of the dynamic system [27]. It is on this basis that our previous works extended the symplectic geometry theory into the analysis of the time series [28-29]. The dimension of a nonlinear dynamic system has been estimated based on symplectic geometry[4]. The symplectic principal component method has been developed to reduce noise in nonlinear systems [12,28]. Subsequently, some researchers has used and furtherly developed the applications of symplectic theory on the time series. The sprinter's surface EMG signals have been evaluated based on symplectic geometry [29]. The determinism characteristics in a time series have been detected by using symplectic geometry method [30]. The symplectic geometry spectrum regression method has been proposed to predict noisy time series [3]. The above research works have shown that the symplectic principal component analysis(SPCA)based methodologies are superior to SVD-based methods for the complex systems. However, little work has been done to quantify how much the disorder, or randomness of a system from the point of view of the symplectic theory.

According to Boltzmann's definition, the interpretation of entropy is a measure of the number of possible microstates of a system. Entropy are used to describe and quantify how much the disorder, uncertainty, or randomness of a system, or the lack of information exist in a system. At present, many entropy estimation approaches, such as Shannon entropy, approximate entropy, sample entropy, fuzzy entropy etc., have been proposed and widely used to quantify the complexity of time series in various fields [31-36]. As a broad and general concept, shannon entropy has been widely used in information theory as well as thermodynamics since Claude Shannon devised in 1948 [37]. This paper presents a symplectic entropy(SymEn) approach based on SPCA and Shannon entropy. The SymEn approach measures the energy distribution of the dynamic system in the symplectic space. To test the utility of the SymEn approach, we choose several typical time series (including the noise data and three chaotic time series) and four kinds of engineering signals(the EEG signals from the brain systems for healthy, ASD subjects, and the sound and vibration signals from the mechanical systems). 


\section{Materials and Methods}

\subsection{Symplectic Entropy}

Given $N$ sampled time series $x_{1}, x_{2}, \cdots, x_{N}$ coming from a system $\boldsymbol{f}(\boldsymbol{x})$, an attractor $\boldsymbol{X}_{m \times d}$ in phase space can be reconstructed by time delay coordinates method:

$$
\boldsymbol{X}=\left[\begin{array}{c}
\bar{X}_{1}^{T} \\
\bar{X}_{2}^{T} \\
\vdots \\
\bar{X}_{m}^{T}
\end{array}\right]=\left[\begin{array}{cccc}
x_{1} & x_{2} & \cdots & x_{d} \\
x_{2} & x_{3} & \cdots & x_{d+1} \\
\vdots & \vdots & \cdots & \vdots \\
x_{m} & x_{m+1} & \cdots & x_{N}
\end{array}\right]
$$

where $d$ is embedding dimension, $m=N-d+1$ is the number of dots in $d$-dimension reconstruction vector, $\overline{\boldsymbol{X}}_{i}^{T},(i=1, \ldots, m)$, denotes a dot in the phase space. According to Takens' embedding theorem, $X$ reflects the characteristics of the system. Then, a Hamilton matrix $M$ of the system $f(x)$ can be built from $X$ :

$$
M=\left(\begin{array}{cc}
A & 0 \\
0 & -A^{T}
\end{array}\right)
$$

where $\boldsymbol{A}=\boldsymbol{X}^{\boldsymbol{T}} \boldsymbol{X}$. Meanwhile, any Hamilton matrix can keep unchanged at symplectic similar transform in symplectic geometry theory. The related proofs refer to the proofs of Appendix A section in the literature [4]. In symplectic geometry, the symplectic similar transform is regular transform. Its essence is dependent on a bilinear antisymmetric nonsingular cross product-symplectic cross product:

$$
[x, y]=\langle x, J y\rangle
$$

where $x$ and $y$ are $2 n$-dimension vectors.

$$
\boldsymbol{J}=\boldsymbol{J}_{2 n}=\left[\begin{array}{cc}
0 & +\boldsymbol{I}_{n} \\
-\boldsymbol{I}_{\boldsymbol{n}} & 0
\end{array}\right]
$$

And there are also some properties as follows:

Theorem 1. The product of sympletcic matrixes is also a symplectic matrix.

Theorem 2. Suppose Household matrix H is:

$$
\boldsymbol{H}=\boldsymbol{H}(\boldsymbol{k}, \omega)=\left(\begin{array}{ll}
\boldsymbol{P} & 0 \\
0 & \boldsymbol{P}
\end{array}\right)
$$

where

$$
\boldsymbol{P}=\boldsymbol{I}_{n}-\frac{2 \varpi \varpi^{*}}{\varpi^{*} \varpi}, \varpi=\left(0, \cdots, 0 ; \omega_{k}, \cdots, \omega_{n}\right)^{T} \neq 0
$$

so, $\boldsymbol{H}$ is symplectic unitary matrix. $\varpi^{*}$ is $\varpi$ conjugate transposition.

Theorem 3. Let $\boldsymbol{S} \in \boldsymbol{C}^{2 \boldsymbol{n} \times 2 \boldsymbol{n}}$ as symplectic matrix, then $\boldsymbol{S}$ can be decomposed as $\boldsymbol{S}=\boldsymbol{Q R}$, where $\boldsymbol{Q}$ is symplectic unitary matrix and $\mathbf{R}$ is upper triangle matrix.

Here, the Household matrix $\boldsymbol{H}$ can be used as the matrix $\boldsymbol{Q}$. Then, the Hamilton matrix $\boldsymbol{M}$ can be transformed into an upper Hessenberg matrix by the Household matrix $\boldsymbol{H}$,

$$
\boldsymbol{H}^{T} M H=\left(\begin{array}{cc}
B & R \\
0 & B^{T}
\end{array}\right)
$$

where $\boldsymbol{B}$ is upper Hessenberg matrix $\left(\boldsymbol{b}_{i j}=0, i>j+1\right)$. Meanwhile, $\boldsymbol{H}$ can be obtained from the matrix $\boldsymbol{A}[4]$. The eigenvalues of the matrix $\boldsymbol{B}$ are given as $\lambda(\boldsymbol{B})=\left\{\mu_{1}, \mu_{2}, \cdots, \mu_{\boldsymbol{d}}\right\}$. The eigenvalues $\boldsymbol{\lambda}(\boldsymbol{A})$ of the matrix $\boldsymbol{A}$ are equal 
to those of the matrix $\boldsymbol{B}$, i.e. $\boldsymbol{\mu}=\lambda(\boldsymbol{B})=\lambda(\boldsymbol{A})$. The eigenvalues $\mu=\left\{\mu_{1}, \mu_{2}, \cdots, \mu_{\boldsymbol{d}}\right\}$ are sorted by descending order, that is,

$$
\mu_{1}>\mu_{2}>\cdots>\mu_{k}>>\mu_{k+1} \geq \cdots \geq \mu_{d}
$$

The $\boldsymbol{\mu}$ values are called the symplectic principal components of $\boldsymbol{A}$ with relevant symplectic orthonormal bases. They reflect the distribution of the energy of the system in different symplectic directions. The probability of the energy distribution at each direction can be defined respectively as $\boldsymbol{p}_{1}, \boldsymbol{p}_{2}, \cdots, \boldsymbol{p}_{\boldsymbol{d}}$ and the probability space of equation can be expressed as:

$$
\begin{gathered}
{[\boldsymbol{M} \bullet \boldsymbol{P}]=\left\{\begin{array}{l}
\boldsymbol{M}: \mu_{1}, \mu_{2}, \cdots, \mu_{\boldsymbol{d}} \\
\boldsymbol{P}: \boldsymbol{p}_{1}, \boldsymbol{p}_{2}, \cdots, \boldsymbol{p}_{\boldsymbol{d}}
\end{array}\right.} \\
\boldsymbol{p}_{i}=\boldsymbol{\mu}_{i} / \sum_{i=1}^{d} \boldsymbol{\mu}_{i}, i=1, \ldots, d
\end{gathered}
$$

where $\boldsymbol{d}$ is the embedding dimension, $\sum_{i=1}^{d} p_{i}=1,0 \leq \boldsymbol{p}_{\boldsymbol{i}} \leq 1$. It represents the uncertainty of the entropy in each direction. Then, an entropy can be defined as follows:

$$
\text { SymEn }=-\sum_{i=1}^{d} \boldsymbol{p}_{i} \log \left(\boldsymbol{p}_{i}\right)
$$

SymEn gives the statistical average and measures the degree of uncertainty of energy about the underlying probability distribution in different directions of a system, in terms of the Shannon entropy, called as Symplectic Entropy.

\subsection{Materials}

A complex system, especially with chaos, is often random-like. To detect nonlinearity of a time series is one of the inverse problems for a complex system, particularly for a chaotic dynamical system. Here, to assess the utility of the SymEn method as a measure for complex systems, the SymEn method is used to test the nonlinearity in the data from both synthetic and real experiments. In section 2.2.1, we illustrate the four synthetic time series from the Gaussian white noise processing and three chaotic systems whose underlying dynamics are known. In section 2.2.2, we consider several real time series from practical systems. In section 2.2.3, we introduce the null hypothesis technique in order to test the difference between the raw data and its surrogate data based on the entropy measures including the SymEn, ApEn, SampEn and FuzzyEn entropy methods.

\subsubsection{Synthetic Time Series}

The In practical systems, noise is generally regarded as the Gaussian white noise having indenpendent and identical probability distribution. Its distribution probability of each direction is even in the dimension $d$ (see Figure 1). The probability value is calculated by Equation 10 . For the equiprobable distribution, the entropy should increase with the number of the embedding dimension d. Here, the Gaussian white noise with mean 0 and variance 1 is used to test if the SymEn estimate is suitable to evaluate the noise. In order to detect if the SymEn method could reflect nonlinear characteristics of a time series, the three typical chaotic systems are applied to generate the corresponding nonlinear time series. 


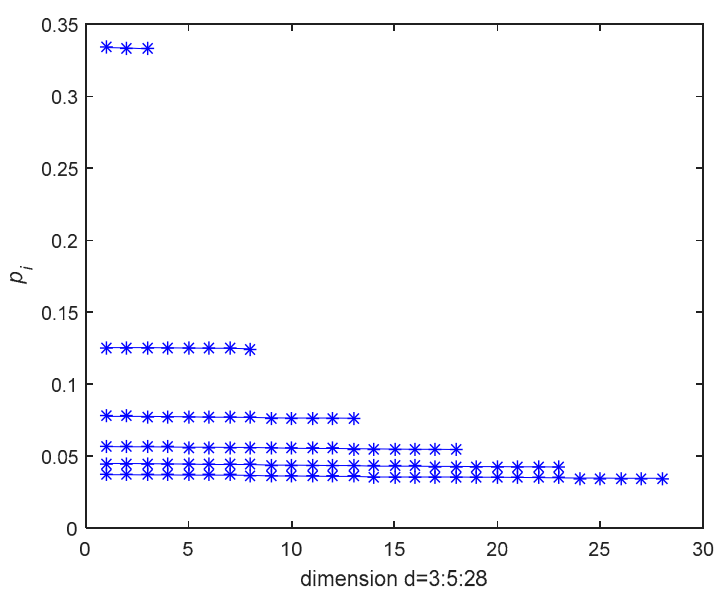

Figure 1. The probability values of the Gaussian white noise in different direction in dimention $\mathrm{d}=3: 5: 28$.

The three chaotic systems are given as follows:

Lorenz chaotic system:

$$
\begin{aligned}
\dot{x} & =10(y-x) \\
\dot{y} & =28 x-y-x z \\
\dot{z} & =x y-\frac{8}{3} z
\end{aligned}
$$

Rössler chaotic system:

$$
\begin{aligned}
& \dot{x}=-y-z \\
& \dot{y}=x+0.15 y \\
& \dot{z}=0.2+z(x-10)
\end{aligned}
$$

Van der Pol chaotic system:

$$
\ddot{\boldsymbol{x}}-5\left(1-\boldsymbol{x}^{2}\right) \dot{\boldsymbol{x}}+\boldsymbol{x}=5 \cos (2.466 \boldsymbol{t})
$$

\subsubsection{Real Time Series}

To further illustrate the application of the SymEn method to the real data sets, we employ several real data sets, including two bioelectric signals (the electroencephalogram (EEG) data from the children with ASD and healthy subjects), and two mechanical signals (the vibration signals of the air compressor and the engine sound signals).

The brain system is extremely complicated. The EEG data are the electrical signal from the brain when the brain is at work. Therefore, the brain function analysis based on EEG have been of enormous interest. A number of researches have also studied the nonlinear behavior of the brain dynamics in the EEG analysis. However, the EEG signals are complicated and random-like. It has been challenging to detect the nonlinear properties of the EEG data [38]. Here, the EEG signals at the right $(\mathrm{O} 2)$ are collected during the resting state with open eyes for the children with ASD and healthy subjects, respectively. The sampling conditions are detailed in previous papers [39-40].

In the mechanical engineering field, there are many complex mechanical systems, such as the air compressor and the car engine [41-42]. Here, the nonlinear characteristics in the abnormal vibration signals of the air compressor and the sound data of the fault diesel engines are detected by the SymEn method. For the air compressor, the vibration acceleration data are collected by the $25,600 \mathrm{~Hz}$ sampling frequency. The length of the measured data is 32,768 points. For the diesel engine, the sound time series are given by the $48 \mathrm{kHz}$ sampling frequency. 


\subsubsection{Surrogate Data and Null Hypothesis}

Surrogate data has been widely employed to discriminate the nonlinear dynamics in real data and typical chaotic time series [36, 43-44]. The idea of surrogate data is to first specify a stochastic process with some linear properties which are consistent with the original data as a null hypothesis, then generate surrogate data sets according to this hypothesis, and calculate a test statistic for the original data and its surrogate data, respectively. If the statistic value of the original data is obviously different from those of its surrogate data, this null hypothesis is rejected and nonlinearity is detected. Here, we apply a linear autocorrelated Gaussian noise process as a null hypothesis. This null hypothesis can generate the surrogate data with the same mean, variance, and spectrum power as the original data. The algorithm of this null hypothesis has been detailed in our early work [44]. In this study, the SymEn measure is used as a test statistic, as well as the the ApEn, SampEn and FuzzyEn measures. In order to give the difference degree between the original data and its surrogate data, the $\mathrm{z}$ value is given:

$$
\boldsymbol{z}=\frac{\left|\boldsymbol{Q}_{\text {orig }}-\left\langle\boldsymbol{Q}_{s}\right\rangle\right|}{\sigma_{s}}
$$

where $\boldsymbol{Q}_{\text {orig }}$ is the test statistic value for the original data, $\left\langle\boldsymbol{Q}_{s}\right\rangle$ is the mean of the statistic values for the surrogates. $\sigma_{s}$ is the standard deviation (SD) of the statistic values for the surrogates. $z>1.96$ means that the null hypothesis can be rejected for two sided testing at $95 \%(\alpha=0.05)$ confidence level. For $\alpha=0.05$, the number of the surrogates is $B=2 / \alpha-1=39[36,38,45]$. For this, 39 sets of the surrogate data are generated for each analyzed data in this study.

Besides, it is necessary to note that the analysis length of each analysis data is 1000 points in this study.

\section{Results}

\subsection{Applicantion to Synthetic Time Series}

In this section, the proposed SymEn method is applied to analyze several synthetic time series (Gaussian white noise and three chaotic time series) in Section 2.2.1. Meanwhile, the reported results are compared with those given by the ApEn, SampEn and FuzzyEn methods.

\subsubsection{Tests on Gaussian White Noise Process}

For the noise data from the Gaussian white noise process, we note that its surrogates which are generated according to the null hypothesis of a linear autocorrelated Gaussian noise process should give negative results. In other words, the null hypothesis should not be rejected. Figure 2 gives the analysis of the noise time series and its surrogate data on the embedding dimension $d=2: 25$ for four entropy methods. The results show that for the different methods, the entropy values are different (see Figure 2). However, there is no difference between the noise and its surrogate data for the four entropy methods. These results indicate that the null hypothesis is accepted in $95 \%$ confidence level. In other words, the noise data is from the same dynamical process as its surrogates from the null hypothesis. Considering that the dynamical property of the noise is equiprobable distribution in different embedding dimension d (see Figure 1), its entropy values should increase with the number of the embedding dimension. The symplectic entropy method presents that the SymEn values of the noise and its surrogates increase with the increase of the embedding dimensions (see Figure 2a). But for the approximate entropy, the values decrease with the increase of the embedding dimensions (see Figure $2 b)$. For the sample entropy, the values vary with the increase of the embedding dimensions, especially no values in the higher dimensions (see Figure 2c). For the fuzzy entropy, with the increase of the dimension, the values decrease a little in the lower dimensions and then increase rapidly in the higher dimensions (see Figure 2d). 


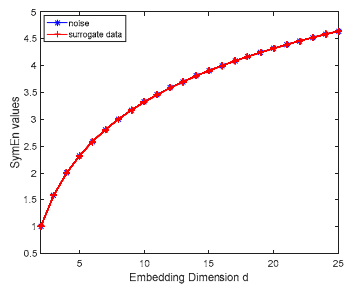

(a) SymEn

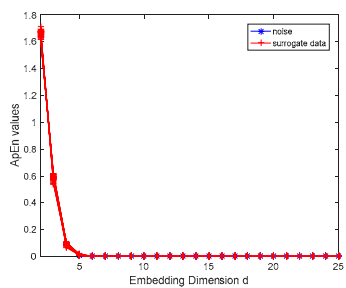

(b) ApEn

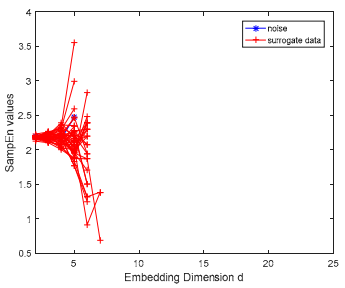

(c) SampEn

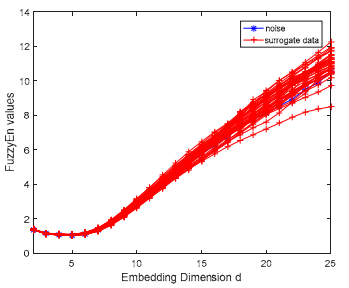

(d) FuzzyEn

Figure 2. The characteristic test of the Gaussian white noise process: (a) the SymEn values of noise and its surrogate data; (b) the ApEn values of noise and its surrogate data; (c) the SampEn values of noise and its surrogate data; (d) the FuzzyEn values of noise and its surrogate data.

\subsubsection{Tests on Chaotic Dynamical Systems}

Three chaotic time series coming from three chaotic dynamical systems are analyzed by the SymEn, ApEn, SampEn and FuzzyEn method, respectively. Figure 3 gives the results of four entropy methods for these time series and their surrogate data. Figure $3 i$ shows that the Lorenz time series is different from its surrogate data in the embedding dimension $d=2: 25$. The SymEn values of the original data increase and tend to be flat with the increase of the embedding dimension while those of its surrogate data only increase. The difference between the original data and its surrogates becomes widened with the increase of the dimension. Moreover, the results of the ApEn, SampEn and FuzzyEn methods are similar to that of the SymEn method although these values are dissimilar for different methods. That is, the entropy values of the raw data are also different from those of its surrogate data for the ApEn, SampEn and FuzzyEn methods. The results indicate that the null hypothesis is rejected in $95 \%$ level for four entropy methods. The Lorenz chaotic time series is not random signal generated from a linear autocorrelated Gaussian process. The dynamical properties of the Lorenz chaotic time series are not the same as the dynamic characteristics of the surrogate data. The raw data should contain some nonlinear components. Furthermore, the chaotic time series from Rössler and Van der Pol chaotic dynamical systems are analyzed by the SymEn, ApEn, SampEn and FuzzyEn methods (see Figure 3ii and 3iii). We can see that there is a significant difference between the entropy values of the raw Rössler and Van der Pol chaotic data and those of their surrogate data, respectively. The null hypothesis is rejected in $95 \%$ confidence level for the raw Rössler and Van der Pol chaotic data, respectively. The results indicate that there are the nonlinear characteristics in the the raw Rössler and Van der Pol chaotic data. By comparison with the ApEn, SampEn and FuzzyEn methods, the SymEn method is able to detect the nonlinearity of the chaotic time series whose the underlying dynamic property is known.

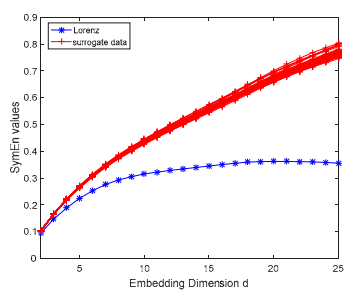

(a) SymEn values

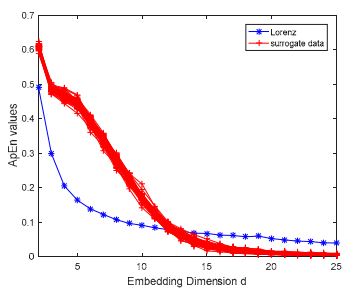

(b) ApEn values

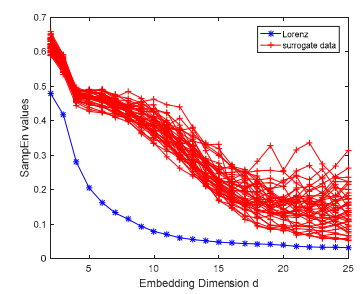

(c) SampEn values

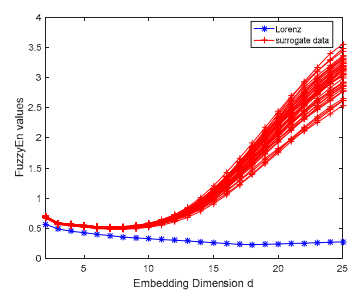

(d) FuzzyEn values

(i) Lorenz chaotic dynamical system 


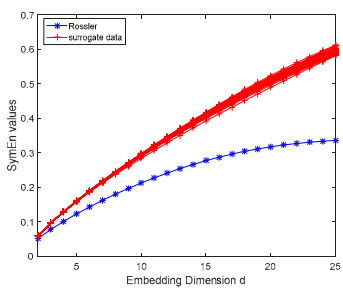

(e) SymEn values

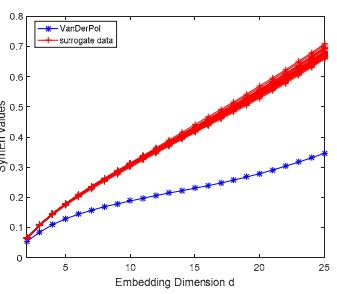

(i) SymEn values

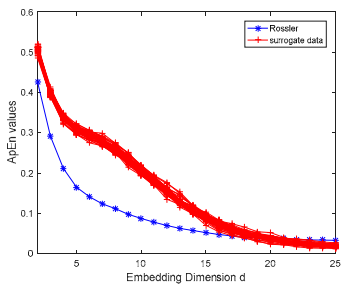

(f) ApEn values

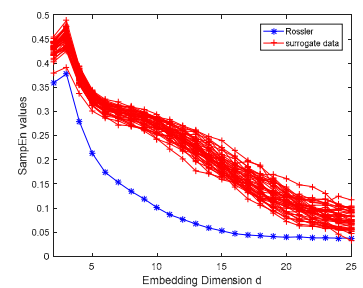

(g) SampEn values

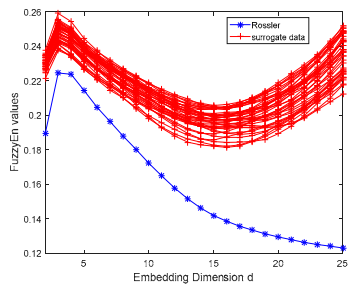

(h) FuzzyEn values

(ii) Rössler chaotic dynamical system

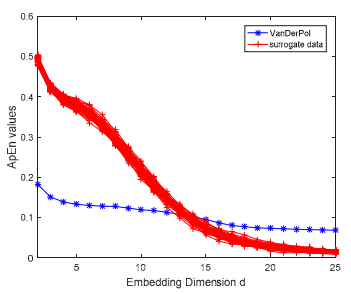

(j) ApEn values

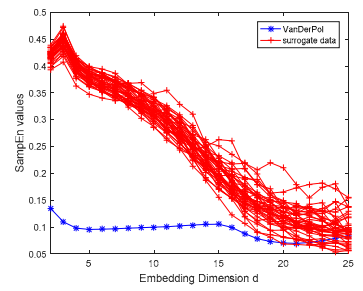

(k) SampEn values

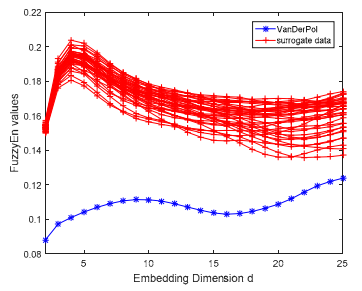

(1) FuzzyEn values

(iii) Van de Pol chaotic dynamical system

Figure 3. Comparison of nonlinear tests on four entropy methods for three chaotic dynamical systems.

\subsection{Applicantion to Real Time Series}

To further test the application of the SymEn method to the real data, some results are reported on experimental time series from several sources. We just attempt to illustrate the SymEn can be regarded as a measure to reflect the dynamic characteristics of time series from real complex systems by comparison with the ApEn, SampEn and FuzzyEn methods.

\subsubsection{The EEG for ASD and Healthy Subjects}

The EEG signal has been used widely to investigate the electrical brain activity. Although a lot of nonlinear measures have been employed to deal with the EEG signals, such as ApEn and SampEn [32,46], the research papers have mostly focused on the classification analysis of EEG signals. But, it is lack to explore the nonlinearity in the EEG data. In particular, few studies detect the nonlinearity of the raw EEG data of ASD. For the children with ASD, some nonlinear measures have applied to directly analyze the corresponding EEG data in the resting in order to explore the brain mechanics of ASD [40]. In this paper, we perform nonlinearity tests based on the SymEn measure on the EEG signals (O2) of ASD and healthy subject, respectively. Figure 4 shows the raw EEG data for ASD subject in resting with open eyes along with the SymEn functions of the raw EEG data and its surrogates. The SymEn values of the raw EEG are distinctly different from those of its surrogates, particularly in the higher dimensions (see Figure $4 b, c)$. The test of the raw EEG data can reject the null hypothesis of a linear stochastic process with 95\% level $(z>1.96)$. Meanwhile, the results of the ApEn, SampEn, and FuzzyEn methods are also shown in Figure 4. For the ApEn method, there is some difference between the raw data and its surrogate data in the low dimensions (see Figure 4d). The discrepancies also exist in the SampEn values of the raw data and its surrogate data in some embedding dimensions (see Figure 4e). The result of the FuzzyEn method is similar to that of the SampEn method (see Figure 4f). However, the differences between the raw data and its surrogates for the ApEn, SampEn and FuzzyEn methods are not more obvious than that for the SymEn method, particularly in the higher embedding dimensions. 


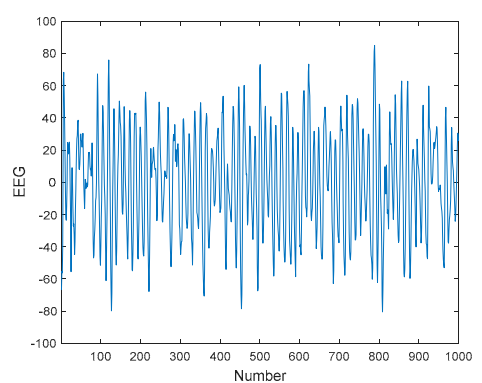

(a)

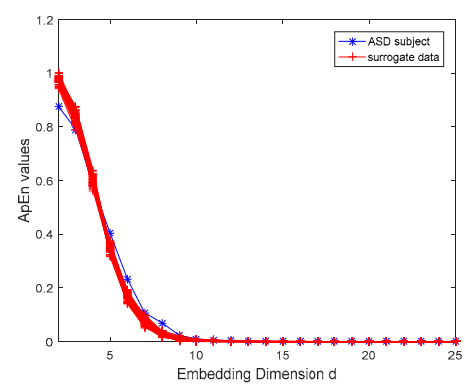

(d) ApEn values

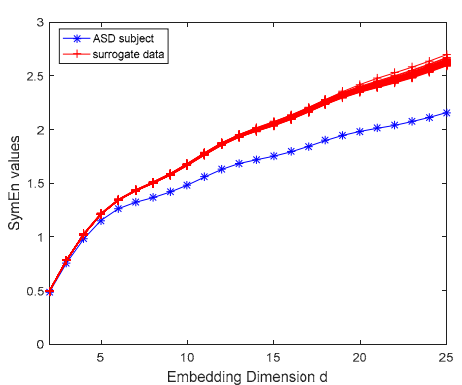

(b)

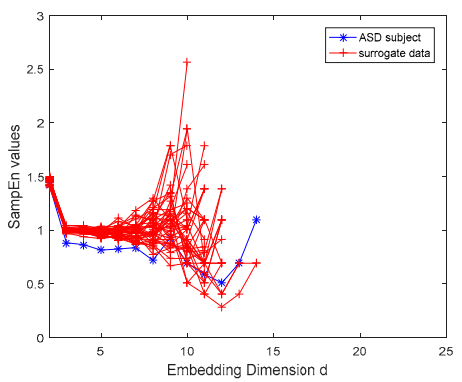

(e) SampEn values

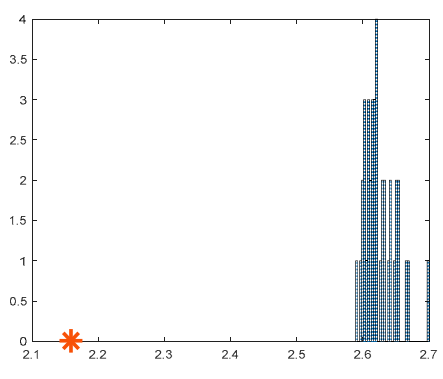

(c)

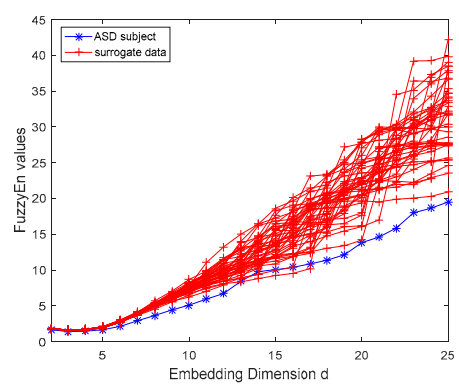

(f) FuzzyEn values

Figure 4. The nonlinearity test on the EEG signal of ASD subject based on the SymEn method by comparison with the ApEn, SampEn and FuzzyEn methods: (a) The raw EEG data in the right O2; (b) The SymEn functions of the raw data and its surrogates in the embedding dimension $d=2: 25$; (c) The SymEn values in $\mathrm{d}=25$ for the raw data and its surrogates, * for the raw data; (d) The ApEn values of the raw data and its surrogates in the embedding dimension $d=2: 25$; (e) The SampEn values of the raw data and its surrogates in the embedding dimension $d=2: 25$; (f) The FuzzyEn values of the raw data and its surrogates in the embedding dimension $\mathrm{d}=2: 25$.

For the healthy subject, the results are similar to those of the ASD subject. Figure 5 presents the raw EEG signal as well as the SymEn, ApEn, SampEn and FuzzyEn measures of the raw data and its surrogates. It can be observed that the SymEn values of the raw data do not belong to the distribution of the SymEn functions of the surrogates (see Figure 5b). Particularly in the dimension $d=25(z>$ 1.96), the test of the raw data rejects hypothesis of belonging to the the same distribution as all of the 39 surrogates (see Figure 5b,c). The result indicates that the raw EEG data contains the nonlinear dynamical properties. The raw EEG data should come from a nonlinear dynamical system. But, the results for the ApEn, SampEn and FuzzyEn methods are not better than that of the SymEn method (see Figure 5b,d-f).

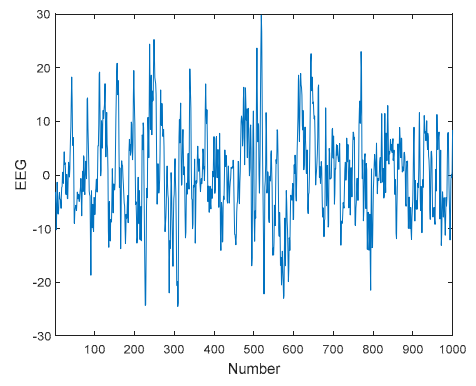

(a)

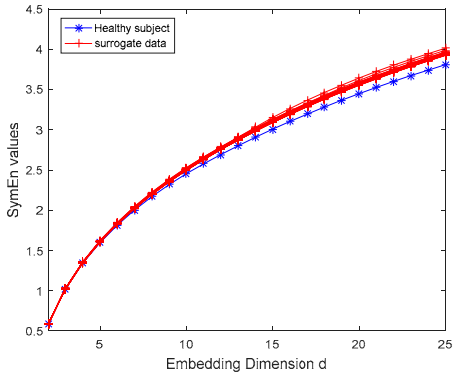

(b)

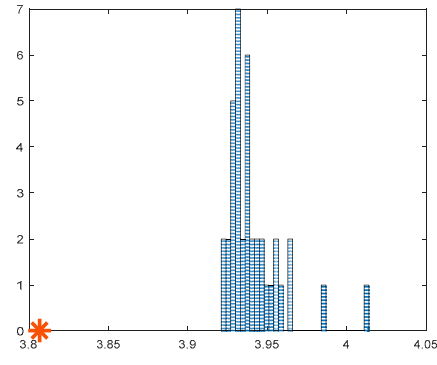

(c) 


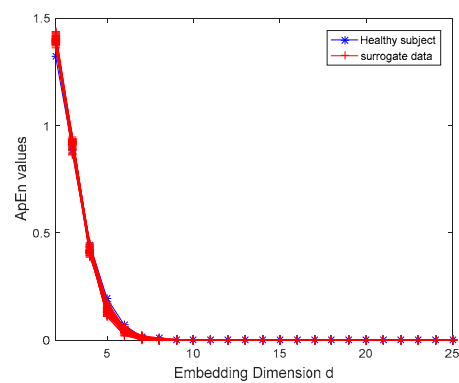

(d) ApEn values

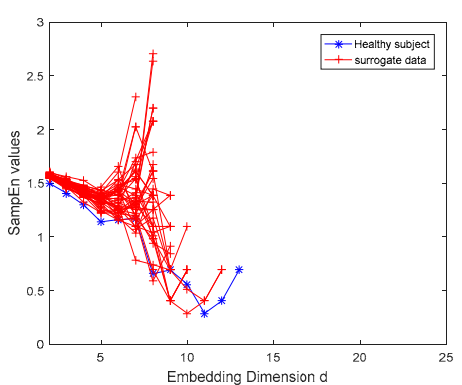

(e) SampEn values

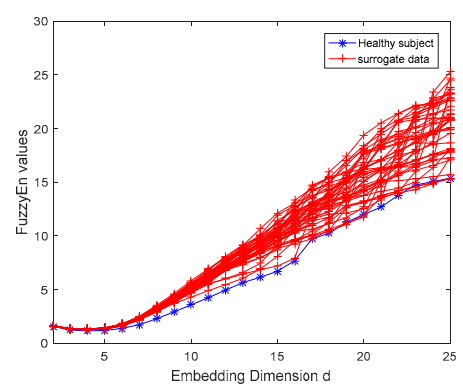

(f) FuzzyEn values

Figure 5. The nonlinearity test on the EEG signal of healthy subject: (a) The raw EEG data in the right O2; (b) In the range of 2 to 25 dimension, the SymEn functions of the raw data and its surrogates; (c) The SymEn measures in $\mathrm{d}=25$ for the raw data and its surrogates; (d) the ApEn values of the raw data and its surrogates in $d=2: 25$; (e) the SampEn values of the raw data and its surrogates in $d=$ $2: 25 ;$ (f) the FuzzyEn values of the raw data and its surrogates in $d=2: 25$.

\subsubsection{The Time Series for Diesel Engine and Air Compressor}

The mechanical systems are also a kind of complex systems, such as diesel engines and air compressors. In engine systems, acoustic signals are often applied to detect the mechanical faults because they can provide significant dynamic information on the engines [42]. Many acoustic methods are employed to deal with engine faults, such as acoustic emission analysis [47]. However, the fault diagnosis based on acoustic signals is often easily affected by background noise [42]. Few studies employ nonlinear analysis methods to explore the characteristics of the abnormal sound in diesel engines. In this paper, we first apply the proposed SymEn function to test the nonlinearity of the abnormal sound of the diesel engine. Figure 6 presents one episode of the original sound data in the fault diesel engine and its SymEn values with those of its surrogates. Our SymEn-based tests applied to the sound data reject the null hypothesis with $95 \%$ confidence over 39 surrogates. In dimension $d=25$, the SymEn value of the original data is obviously different from those of its surrogates with the difference degree $z=16.99(z>1.96)$. The results show that the dynamical characteristics of the raw sound data are not linear. The raw data should come from a nonlinear system. Meanwhile, the ApEn, SampEn and FuzzyEn methods are also employed to analyze the sound data (see Figure $6 \mathrm{~d}-\mathrm{f}$ ). In general, the results are worse than that of the SymEn method, although the ApEn values show some difference between the raw data and its surrogate in $d=3,4$.

Finally, we exmine the vibration acceleration data in the air compressor and its surrogates. Air compressors are extensively employed mechanical systems. The working state of the air compressor becomes unstable due to the interaction of pistons, the pressure instability of the compressed air outputted from the air compressor in volatile gas-consumption situations. Besides, the environment noise has often a strong impact on the vibration measurement. Hence, the measured vibration signals often show the complex dynamics behaviors. This paper considers the vibration acceleration collected at the fault state of the air compressor. Figure 7 gives the original series and its SymEn function as well as those of its surrogate data. The SymEn of the raw data is obviously distinguishable from those of its surrogates. The null hypothesis is rejected at $95 \%$ significance level, especially in $\mathrm{d}$ $=25(z>1.96)$. The raw data is not from a linear process in the null hypothesis. The result indicates that the raw data is nonlinear. Besides, the ApEn, SampEn and FuzzyEn methods are also used to analyze the raw vibration data (see Figure $7 \mathrm{~d}-\mathrm{f}$ ). The results of the ApEn, SampEn and FuzzyEn are worse than those of the SymEn method. 


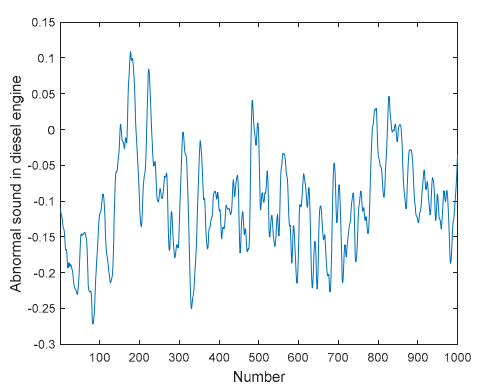

(a)

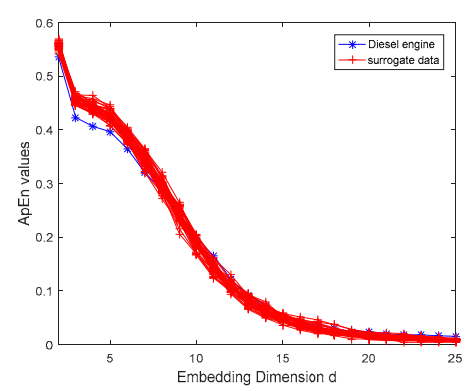

(d) ApEn values

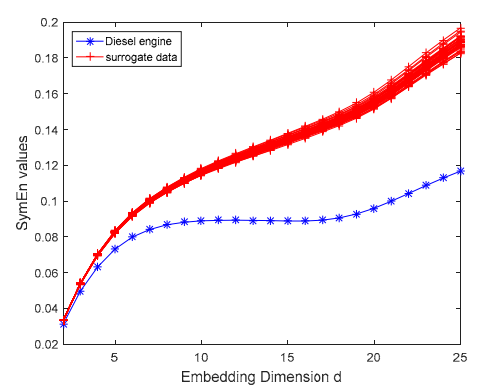

(b)

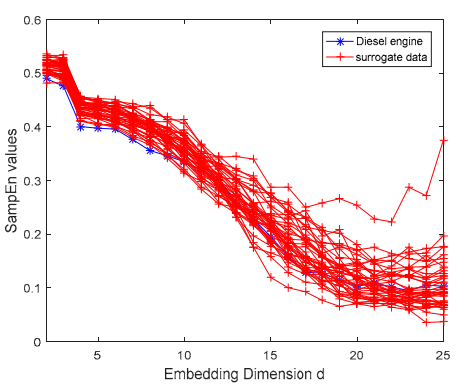

(e) SampEn values

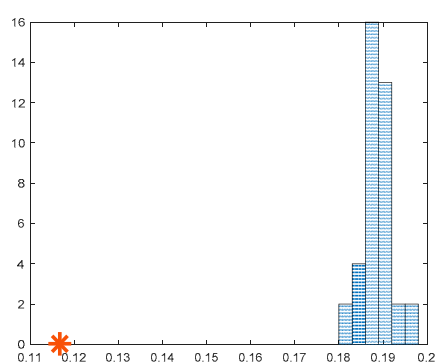

(c)

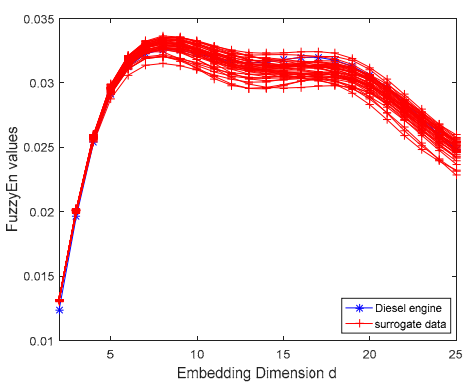

(f) FuzzyEn values

Figure 6. The test on the abnormal sound in the diesel engine: (a) The raw 1000-point sound time series; (b) The SymEn functions of the raw data and its surrogates in d = 2:25; (c) In d = 25, the SymEn measures of the raw data and its surrogates, * for the raw data, histogram for its surrogates; (d) the ApEn values of the raw data and its surrogates in $d=2: 25$; (e) the SampEn values of the raw data and its surrogates in $\mathrm{d}=2: 25$; (f) the FuzzyEn values of the raw data and its surrogates in $d=2: 25$.

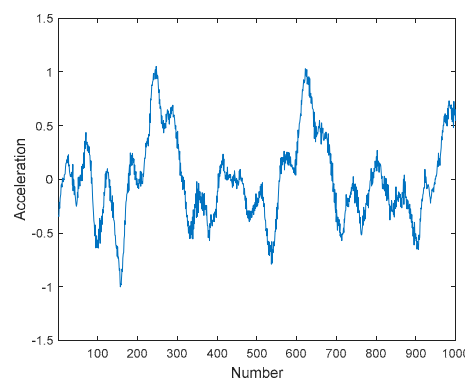

(a)

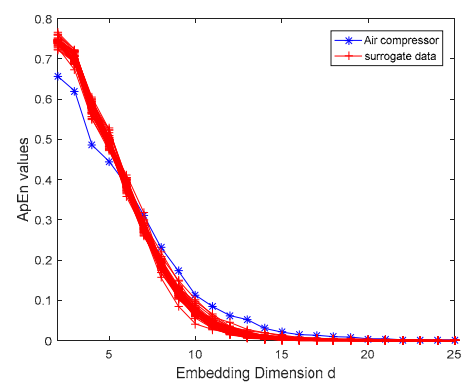

(d) ApEn values

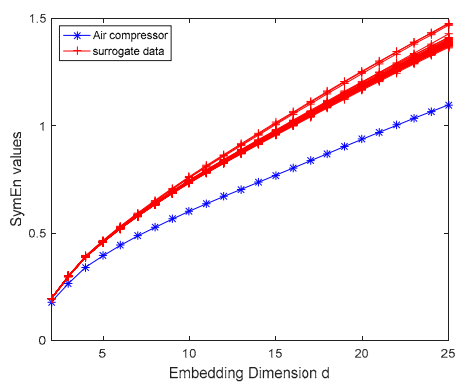

(b)

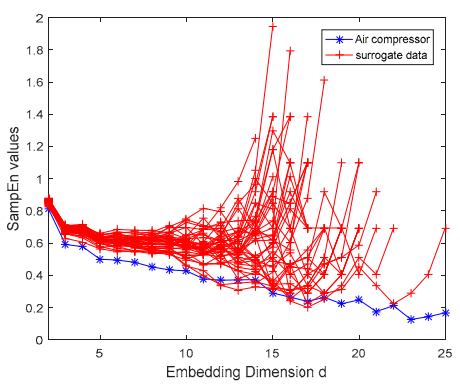

(e) SampEn values

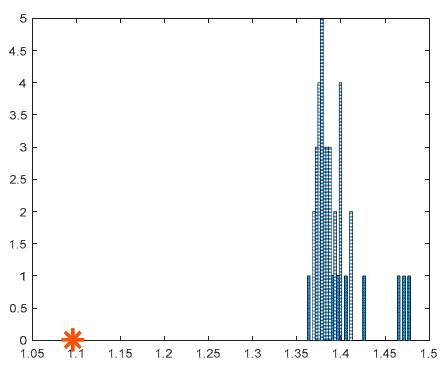

(c)

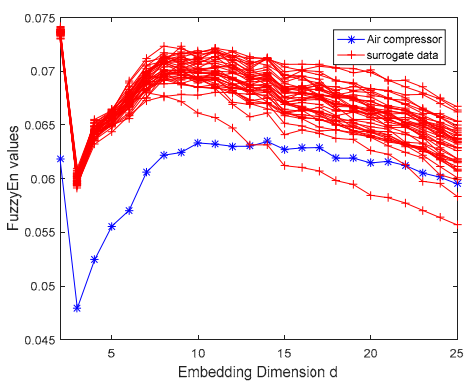

(f) FuzzyEn values

Figure 7. The test on the acceleration time series in the vibration of the air compressor: (a) The raw acceleration signal; (b) The SymEn measures in $d=2: 25$ for the raw data and its surrogates; (c) The SymEn measure ${ }^{*}$ ) of the raw data in $d=25$ and the histogram of the SymEn values for its surrogates; 
(d) the ApEn values of the raw data and its surrogates in $d=2: 25$; (e) the SampEn values of the raw data and its surrogates in $d=2: 25$; (f) the FuzzyEn values of the raw data and its surrogates in $d=$ $2: 25$.

From the above analysis, the results have shown that the proposed SymEn function could be a suitable measure for nonlinearity tests, not only on synthetic data, but also on the limited samples of noisy measurements from real world systems.

\section{Discussion}

We propose the SymEn function method and test four types of synthetic time series. And for the Gaussian white noise, its distribution is equiprobable. The probability distribution in the SymEn function is equal for the noise (see Figure 1). The SymEn method reflects the distribution of the noise data efficaciously. For the typical chaotic time series, the SymEn function method gives the differences between the raw chaotic data and its surrogate data (see Figure 3). According to the null hypothesis, the test on chaotic time series with the SymEn function favors the rejection of the null hypothesis. The results are consistent with those of the ApEn, SampEn and FuzzyEn functions. As can be seen, the SymEn measure generally perform properly for the wider range of the embedding dimensions. The ApEn and SampEn measures turn out to be unable to discriminate a chaotic system from its surrogate data sets in higher embedding dimensions. The FuzzyEn function is better than them but worse than the SymEn.

For the nonlinearity test of the EEG signals, previous studies had found that the nonlinear results were varied for the different methods and the EEG at the different brain areas $[43,48]$. Theiler et al. [43] found that the EEG data at the left central(C3) could reject the null hypothesis of a linear stochastic process whereas other any EEG data (O1) could not. Kugiumtzis also pointed out that cross-examing the results with other methods seemed to be necessary [48]. To examine the practicability of the SymEn function method, the difference degree $z$ values (referring to Equation 15) are further given in Figure 8 for the four entropy methods to test the EEG signals of the ASD and healthy subjects. For the suitable range of the embedding dimension (such as $d=5$ ), the tests on the EEG signals are consistent for the four entropy methods (see Figure 8). For the ApEn, SampEn and FuzzyEn, variations of the difference degrees are shown with the different embedding dimensions. But, the SymEn method performs better than the other three methods. For the embedding dimension $\mathrm{d}=2: 25$, all the $\mathrm{z}$ values of the SymEn function are larger than 1.96 . The rejection can be obtained at the wide range of the embedding dimension for the SymEn method. The EEG data can come from a nonlinear brain system. The results are consistent with those in the previous literatures $[43,48]$.

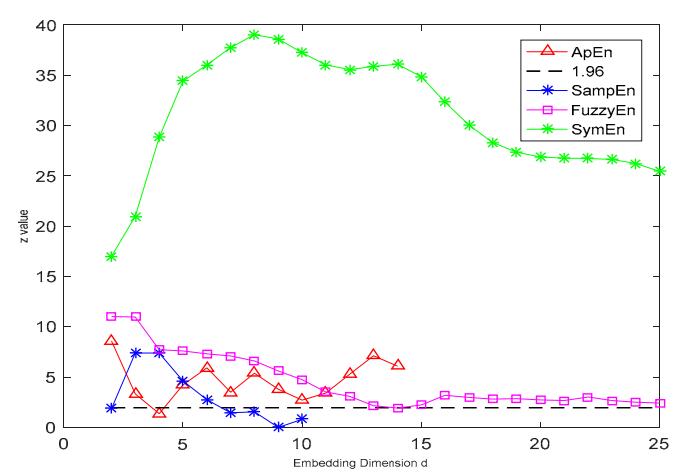

(a) ASD subject

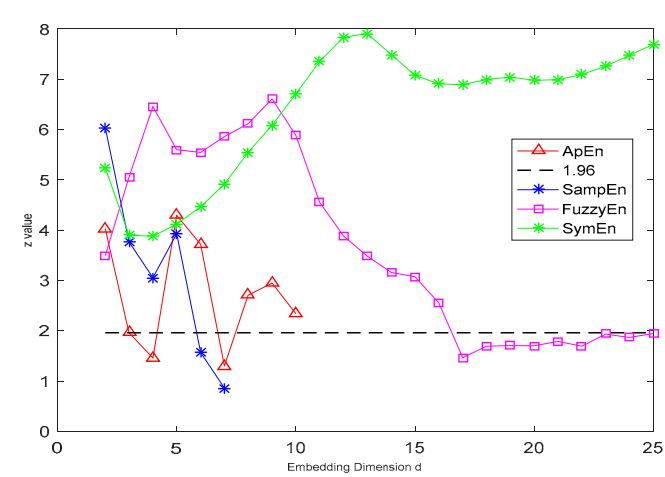

(b) Healthy subject

Figure 8. The difference degree $\mathrm{z}$ values of the entropy values in the Figure 4 and Figure 5 for four entropy methods.

For the sound and vibration signals, we further to exhibit the the difference degree $\mathrm{z}$ values of the discrimination between the original data and its surrogate data in Figure 9. The ApEn, SampEn and FuzzyEn methods do not achieve all of the difference degrees are larger than 1.96 for all 
$\mathrm{d}=2$ :25. But for some embedding dimensions, such as $\mathrm{d}=3$ or 4 , the four methods give consistent discriminations for the sound and vibration signals(see Figure $9 a, b$ ). The results suggest the rejection of the null hypothesis. And for the SymEn method, the performance of the test on the different embedding dimensions is uniform in $d=2: 25$. The results indicate that the SymEn method are better than the ApEn, SampEn and FuzzyEn methods.

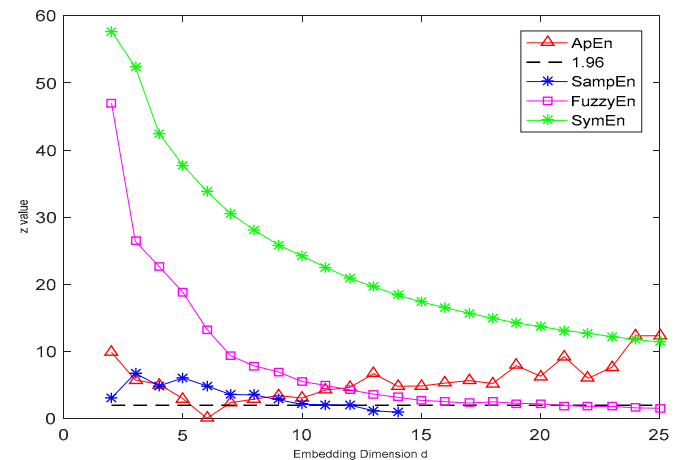

(a) Air compressor

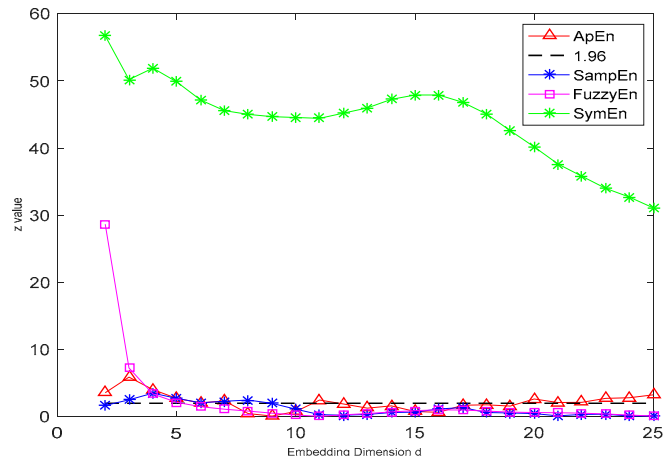

(b) Diesel engine

Figure 9. The difference degree $z$ values of the entropy values in Figure 6 and Figure 7 for the four entropy methods.

\section{Conclusions}

In this paper, the symplectic entropy (SymEn) measure has been proposed to measure the disorder of the system. Its algorithm is a logarithmic measure of the average amount of energy about the underlying probability distribution in different directions of a system, like the Shannon entropy. The higher the disorder of the system is, the higher the entropy. For a Gaussion white noise process, the SymEn method reflect this point because the SymEn values become larger with the increase of the embedding dimension. Meanwhile, we study the effectiveness and practicability of the SymEn method experimentally by testing the nonlinearity of the synthetic chaotic data and real time series. In comparison with the ApEn, SampEn and FuzzyEn entropy measures, the results show that the performance of the proposed SymEn method is superior to them. The SymEn method can reflect the characteritics of the systems and can be used to detect if there are the nonlinear components in the real data from a complex system.

Acknowledgments: We would like to thank three anonymous reviewers for their helpful comments on this article. We are pleased to acknowledge Xie Hongbo for useful discussions to improve this paper. And we also wish to thank Fan Jing and Bian Dayi in Vanderbilt University for experiments. This work was partially supported by the Science Fund for Creative Research Groups of the National Natural Science Foundation of China (Grant No. 51421092), the National Natural Science Foundation of China (Grant No. 10872125), the State Scholarship Fund of China Scholarship Council (Grant No. [2013]3018), Research Fund of State Key Laboratory of Mechanical System and Vibration (Grant No. MSV-MS-2010-08), Research Fund from Shanghai Jiao Tong University for medical and engineering science. (Grant No. YG2013MS74), the NSF Project of USA (Grant Nos. 0967170, 1264462), and the NIH project of USA (Grant Nos. 1R01MH091102-01A1, 1R21MH103518-01).

Author Contributions: Min Lei was in charge of framing the concept and method, developing the program codes, and writing the paper. Guang Meng was in charge of the supervision of this research and helping in revising. Nilanjan Sarkar was in charge of conducting the experiments and supervising this research. Wenming Zhang was in charge of guiding in writing and conducting in revising. Joshua Wade was responsible for the experimental design and data extraction.

Conflicts of Interest: The authors declare no conflict of interest.

\section{References}

1. Consolini, G.; Tozzi, R.; Michelis, P. De. Complexity in the sunspot cycle. Astronomy E Astrophysics 2009, 506, 1381-1391. 
2. Gao, J.B.; Hu, J.; Tung, W.W. Complexity measures of brain wave dynamics. Cognitive Neurodynamics 2011, 5(2), 171-182.

3. Xie, H.; Dokos, S.; Sivakumar, B.; Mengersen, K. Symplectic geometry spectrum regression for prediction of noisy time series. Phyiscal Review E, 2016, 93, 052217.

4. Lei, M., Wang, ZH, Feng, ZJ, A method of embedding dimension estimation based on symplectic geometry. Physics Letters A 2002, 303(2-3), 179-189.

5. Nazimuddin, A. K. M.; Hasan, M. R. Applications of Riemannian geometry comparing with symplectic geometry. Annals of Pure and Applied Mathematics 2014, 6(2), 170-177.

6. Hou, X.H.; Deng Z.C.; Zhou, J.X. Symplectic analysis for the wave propagation properties of conventional and auxetic cellular structures. International Journal of Numerical Analysis and Modelling 2011, 2(4), 298-314.

7. Hong, S.; Kim, Y.; Park, Y.; Rothe, K. D. Symplectic embedding and Hamilton-Jacobi analysis of proca model. Modern Physics Letters A 2002, 17(8), 435-451.

8. Gao, Q.; Tan, S. J.; Zhang, H. W.; Zhong, W. X. Symplectic algorithms based on the principle of least action and generating functions. International Journal for Numerical Methods in Engineering 2012, 89, 438-508.

9. Lim, C.W.; Xu, X.S. Symplectic elasticity: theory and applications. Applied Mechanics Reviews 2010, .63, 050802-1-10.

10. Torres, D.M. Nonlinear symplectic grassmannians and Hamiltonian actions in prequantum line bundles. International Journal of Geometric Methods in Modern Physics 2012, 9(1), 1250001-1-18.

11. Feng, K., 1985, “On Difference Schemes and Symplectic Geometry,” Proceedings of Beijing Symposium Differential Geometry and Differential Equations,

12. Lei, M.; Meng, G. Symplectic principal component analysis: a new method for time series analysis. Mathematical Problems in Engineering 2011, 2011, 793429..

13. Loan, C.,VanAsymplectic method for approximating all the eigenvalues of a Hamiltonian matrix. Linear Algebra Appl. 1984, 61, 233-251.

14. Benner, P. and Faßbender, H., An implicitly restarted symplectic Lanczos method for the Hamiltonian eigenvalue problem, Linear Algebra Appl. 1997, 263, 75-111.

15. Salam, A., Al-Aidarous, E. and Farouk A., EI optimal symplectic householder transformations for sr decomposition, Linear Algebra Appl. 2008, 429, 1334-1353.

16. Salam, A., EI Farouk, A. and Al-Aidarous, E., Symplectic Householder transformations for a QR-like decomposition, a geometric and algebraic approaches, J. Comput. Appl. Math. , 2008, 214, 533-548.

17. Salam, A., On theoretical and numerical aspects of symplectic Gram-Schmidt-like algorithms, Numer. Algorithms, 2005, 39, 237-242.

18. Zhong, W. X., The inverse iteration method for the eigenproblem of large symplectic matrices, Computational Structural Mechanics and Applications,1992, 9( 3), 227-238.

19. Zhong, W.X.; Williams, F.W.; Leung, A.Y.T. Symplectic analsyis for periodical electro-magnetic waveguides. Journal of Sound and Vibration 2003, 267, 227-244.

20. Zhong, W., Plane elasticity problem in strip domain and Hamiltonian system, Journal of Dalian University of Technology 1991, 31(4), 373-384, (in Chinese).

21. Yao, W., Zhong, W. and Lim, C.W., Symplectic Elasticity. World Scientific, 2009, ISBN 978-981-277-870-3, 981-277-870-5.

22. LÜ, C. F., Lim, C. W. AND Yao, W. A., A new analytic symplectic elasticity approach for beams resting on Pasternak elastic foundations. Journal of Mechanics of Materials and Structures, 2009, 4(10), 1741-1754.

23. Zhong, W. X., 2004, Duality System in Applied Mechanics and Optimal Control, Kluwer Academic, Boston.

24. Zhao, L., and Chen, W. Q. Symplectic Analysis of Plane Problems of Functionally Graded Piezoelectric Materials. Mech. Mater. , 2009, 41, 1330-1339.

25. Lim, C.W., Yao, W.A., and Cui, S. Benchmarks of analytical symplectic solutions for bending of cornersupported rectangular thin plates. IES Journal Part A: Civil and Structural Engineering, 2008., 1(2), 106115.

26. Batista, M.. Discussion of 'Benchmark symplectic solutions for bending of corner-supported rectangular thin plates'. IES Journal Part A: Civil and Structural Engineering, 2010, 3(1), 70.

27. Takens, F., Detecting Strange Attractors in Turbulence. In: Dynamical Systems and Turbulence, eds. D. A. Rand and L. -S. Young, Lecture Notes in Mathematics 1981, 898 , 366-381.

28. Lei, M.; Meng, G. A Noise Reduction Method for Continuous Chaotic Systems Based on Symplectic Geometry. Journal of Vibration Engineering \& Technologies, 2015, 3(1), 13-24. 
29. Niu, X., Qu, F., Wang, N., Evaluating Sprinters' Surface EMG Signals Based on EMD and Symplectic Geometry. Journal of Ocean University of Qingdao, vol.35, no.1, pp125-129, 2005. (in Chinese)

30. Xie, H., Wang, Z., Huang, H., Identification determinism in time series based on symplectic geometry spectra. Physics Letters A, vol.342, no.1-2, pp156-161, 2005.

31. Pincus, S.M. Approximate entropy as a measure of system complexity. Proc. Natl. Acad. Sci. 1991, 88, 22972301.

32. Richman, J., Moorman, J. Physiological time-series analysis using approximate entropy and sample entropy. Am. J. Physiol. Heart Circ. Physiol. 2000, 278,H2039-H2049.

33. Guzmán-Vargas, L., Ramírez-Rojas, A., Angulo-Brown, F. Multiscale entropy analysis of electroseismic time series." Nat. Hazards Earth Syst. Sci. 2008, 8, 855-860.

34. Hino, H.; Wakayama K.; Murata, N. Entropy-based sliced inverse regression. Computational Statistics and Data Analysis 2013, 67, 105-114.

35. Elton, R. Johny; Vasuki, P.; Mohanalin, J. Voice Activity Detection Using Fuzzy Entropy and Support Vector Machine. Entropy 2016, 18(8), 298-1-14.

36. Xie, H.; Guo, J.; Zheng, Y. Using the modified sample entropy to detect determinism. Physics Letters A 2010, 374, 3926-3931.

37. Kanner L. Autistic disturbances of affective contact. Nervous Child. 1943, 2, 217-250.

38. Theiler, J.; Prichard D. Constrained-realization Monte-Carlo method for hypothesis testing. Physica D, 1996, 94, 221-235.

39. Lei M, Meng G, Fan J, Wade J, Bian D, Sarkar N. Nonlinear analysis of electroencephalograms of healthy people during driving test based on symplectic principal component analysis method. 2014 24th Annual International Conference of Society for Chaos Theory in Psychology E Life Sciences, Milwaukee, Wisconsin, USA, 31 July - 2 August 2014, p10.

40. Lei M.; Meng G.; Zhang W.M; Sarkar, N. Sample entropy of electroencephalogram for children with autism based on virtual driving game. Acta Physica Sinica 2016, 65, 108701.

41. Rens, J.; Clark, R.E.; Howe, D. Vibration analysis and control of reciprocating air-compressors. International Journal of Applied Electromagnetics and Mechanics 2001/2002, 15, 155-162.

42. Ning, D.; Sun, C.; Gong, Y.; Zhang, Z.; Hou, J. Extraction of fault component from abnormal sound in diesel engines using acoustic signals. Mechanical Systems and Signal Processing 2016, 75, 544-555.

43. Theiler, J.; Eubank, S.; Longtin, A.; Galdrikian, B. Testing for nonlinearity in time series: the method of surrogate data. Physica D, 1992, 58, 77-94.

44. Lei, M.; Wang, Z.; Feng, Z. Detecting nonlinearity of action surface EMG signal. Phys. Lett. A 2001, 290(56), 297-303.

45. Schreiber, T.; Schmitz, A. Discrimination power of measures for nonlinearity in a time series. Physical Review E 1997, 55(5), 5443-5447.

46. Keller, K.; Unakafov, A.M.; Unakafova, V.A. Ordinal patterns, entropy, and EEG. Entropy 2014, 16, 62126239.

47. Muñoz, M.; Moreno, F.; Bermal, N.; Arroyo, J. and Paniagua L. Engine diagnosis method based on vibration and acoustic emission energy. Insight 2012, 54 (3), 149-154

48. Kugiumtzis, D. On the reliability of the surrogate data test for nonlinearity in the analysis of noisy time series. International Journal of Bifurcation and Chaos 2001, 11(7), 1881-1896.

(C) 2016 by the authors; licensee Preprints, Basel, Switzerland. This article is an open access article distributed under the terms and conditions of the Creative Commons by Attribution (CC-BY) license (http://creativecommons.org/licenses/by/4.0/). 